\section{Endoscopic ultrasound-guided biliary drainage with a novel fine-gauge balloon catheter: one-step placement technique}

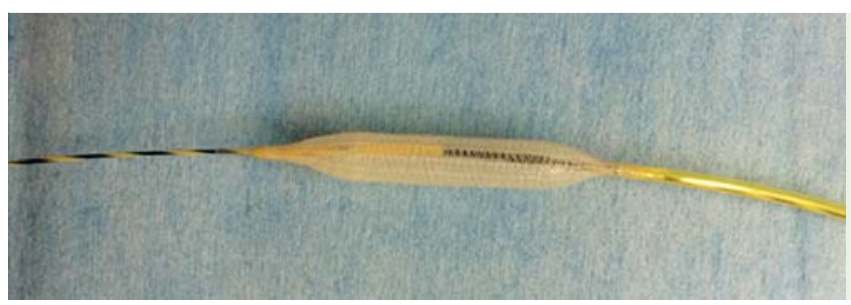

Fig. 1 The tip of this balloon catheter is only $3 \mathrm{Fr}$ in diameter and tapered. The surface of the balloon catheter is rough.
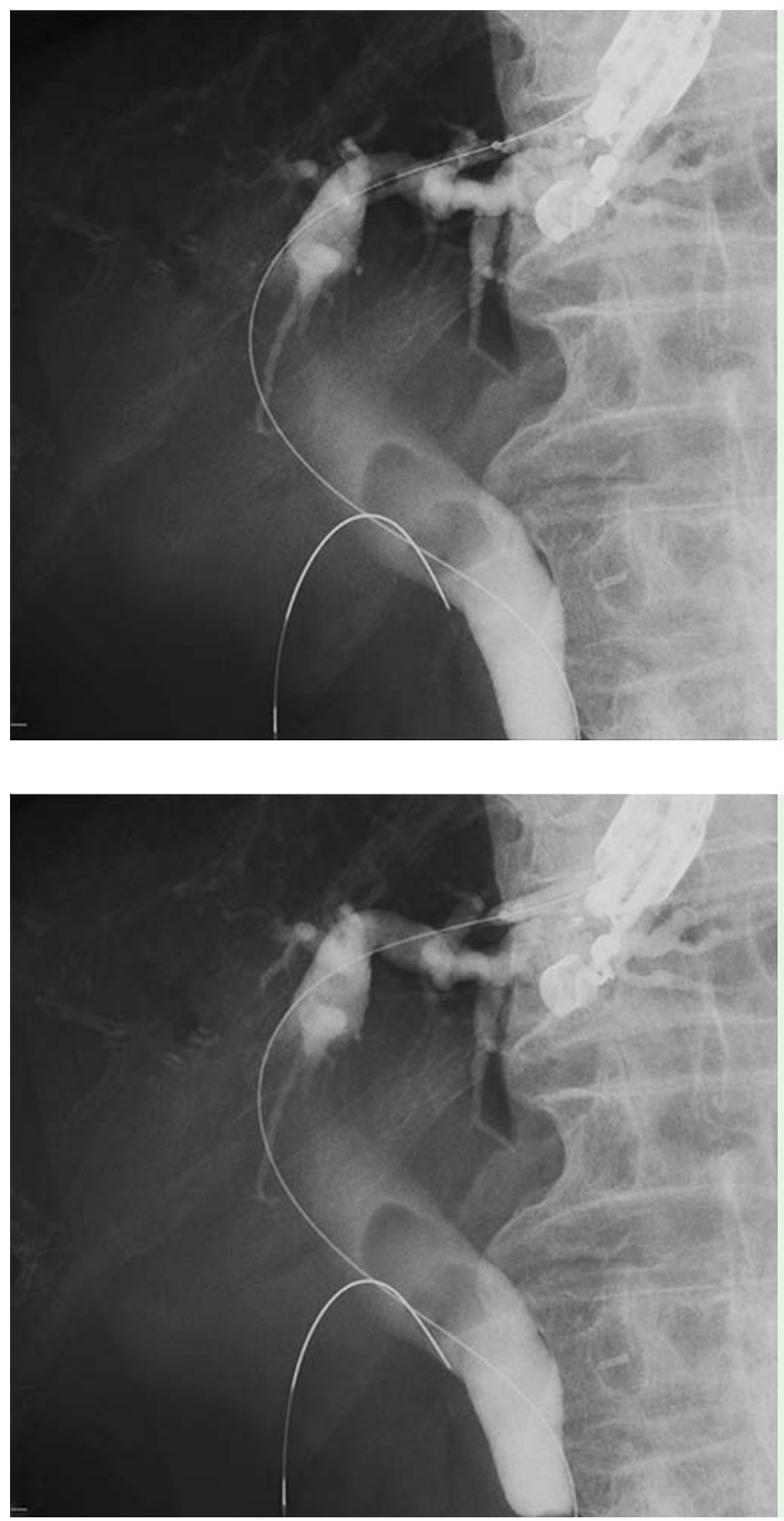

During endoscopic ultrasound-guided hepaticogastrostomy (EUS-HGS), it is necessary to dilate the fistula [1]. Graded dilation with a 4-Fr cannula and 6- and 7-Fr bougie dilators is relatively easily performed, and this stepwise procedure appears to be safe [2]. On the other hand, the use of a needle knife for fistula dilation in endoscopic ultrasound-guided biliary drainage (EUS-BD) may be associated with post-procedural adverse events [3]. These procedures have the potential to cause bile leakage because only the guidewire is placed between the bile duct and intestine across the abdominal cavity during the exchange of devices.

There is a great need for a simplified technique to avoid such adverse events. A novel balloon catheter (ZARA; Century Medical, Tokyo, Japan) has two unique characteristics ( $\bullet$ Fig. 1 ). First, the tip of the balloon catheter is only $3 \mathrm{Fr}$ in diameter and tapered. Second, the surface of the balloon catheter is rough. Therefore, the device is easily inserted following puncture with a 19-gauge needle, and during balloon dilation, the characteristics of the surface may be of clinical benefit in preventing malposition of the dilation site.

An 80-year-old man was admitted because of vomiting and obstructive jaundice due to unresectable pancreatic cancer, which was obstructing the duodenum. First, we performed duodenal stenting. Next, we punctured the intrahepatic bile duct with a 19-gauge fine-needle aspiration needle and inserted a 0.025 -inch stiff guidewire. The novel balloon catheter could then be smoothly inserted into the duodenum across the stricture site and duodenal stent. We dilated the stricture and duodenal stent to place the metallic stent, after which we used the novel balloon catheter to dilate the intrahepatic bile duct ( $\bullet$ Fig.2) and stomach wall ( Fig.3). A metallic stent delivery system was then inserted in antegrade fashion and successfully placed at the lower bile duct. Finally, we performed the EUS-HGS ( $\bullet$ Fig.4). No adverse events occurred.

\section{Video 1}

Contrast medium is injected into the bile duct through a 19-gauge needle, and a 0.025 -inch guidewire is inserted. Then, the novel balloon catheter can easily be inserted, and the ampulla, intrahepatic duct wall, and gastric wall are dilated. Antegrade stenting is done first, followed by endoscopic ultrasound-guided hepaticogastrostomy. 


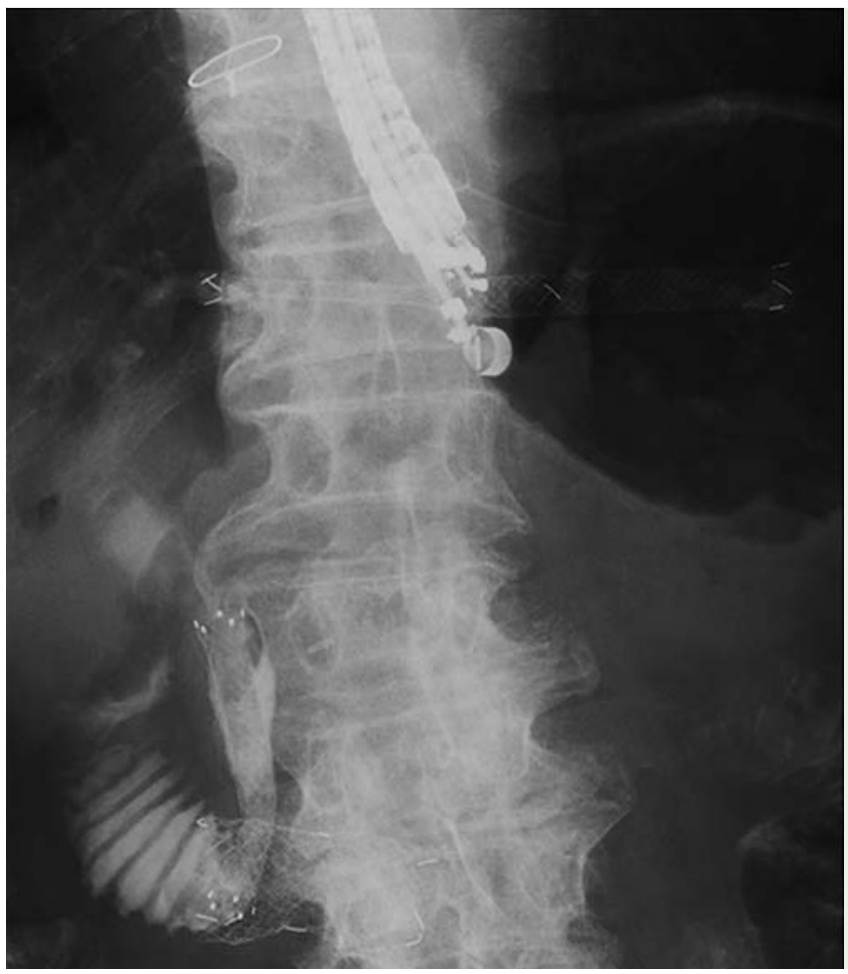

Fig. 4 Successful performance of endoscopic ultrasound-guided hepaticogastrostomy in combination with antegrade stenting.

The duration of the procedure, shown in - Video 1, was only 16 minutes.

This novel balloon catheter may be useful with fistula dilation devices, although a prospective clinical study is needed.

Endoscopy_UCTN_Code_TTT_1AR_2AK
Competing interests: None

Takeshi Ogura, Tatsushi Sano,

Saori Onda, Daisuke Masuda, Akira Imoto, Kazuh de Higuchi

2nd Department of Internal Medicine, Osaka Medical College, Osaka, Japan

\section{References}

1 Park DH. Endoscopic ultrasonography-guided hepaticogastrostomy. Gastrointest Endosc Clin N Am 2012; 22: 271 -280, ix

2 Park DH, Jeong SU, Lee BU et al. Prospective evaluation of a treatment algorithm with enhanced guidewire manipulation protocol for EUS-guided biliary drainage after failed ERCP (with video). Gastrointest Endosc 2013; 78: 91 - 101

3 Park DH, Jeong SU, Lee BU et al. EUS-guided biliary drainage with transluminal stenting after failed ERCP: predictors of adverse events and long-term results. Gastrointest Endosc 2011; 74: 1276-1284

\section{Bibliography}

DOI http://dx.doi.org/

10.1055/s-0034-1391859

Endoscopy 2015; 47: E245-E246

(c) Georg Thieme Verlag KG

Stuttgart · New York

ISSN 0013-726X

\section{Corresponding author \\ Takeshi Ogura, PhD, MD}

2nd Department of Internal Medicine

Osaka Medical College

2-7 Daigakuchou, Takatsukishi

Osaka 569-8686

Japan

Fax: +81-527635233

oguratakeshi0411@yahoo.co.jp 